International Journal of Engineering \& Technology, $7(3.34)(2018) 110-113$
International Journal of Engineering \& Technology
WPC
Website: www.sciencepubco.com/index.php/IJET
Research paper

\title{
Maximum Accuracy in MANET-Node-Localization Using Multi-Stage Trackerwith Less Time-Complexity
}

\author{
Arul selvan.M ${ }^{1}$, Dr.SelvaKumar. $S^{2}$ \\ ${ }^{1}$ Research Scholar, Department of CSC, \\ Bharath Institute of Higher Education and Research, \\ Chennai, India ${ }^{2}$ Professor, Department of Computer Science \& Engineering, \\ GKM College of Engineering \& Technology, Chennai-600 063, India

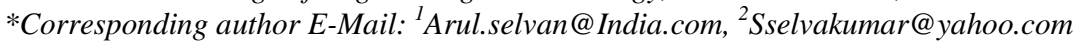

\begin{abstract}
An ad hoc network (referred as MANET), is also called as mobile ad-hoc network and also wireless ad hoc network, is automatic selfconfiguring, infrastructure-less wireless network of mobile devices, and it widely used in various areas. Wireless sensor network composed of wireless sensor nodes and these nodes can do processing, sensing, computation and wireless communication. Because of its more functionality and low cost of energy consumption the wireless sensors are used widely in various areas such as ranging from sensors for environment, road safety, home, peer-to-peer messaging, vehicular ad hoc communications, health, disaster recovery operations, robots, weapons, defense, etc. The localization information is very important for the operation of MANET because of security, routing, and other needs. This articles mainly contracts with the network node station identification in wireless environment with maximum accuracy. There are many methods of localization exist for MANET, and this paper discusses, compares and explains the betterments through experimental localization methods. Localization process is a vital and essential part in lots of applications that signifies a supplementary procedure in a network function or sometime it can be a primary goal of the application. Localization, as an essential procedure is also desirable based on data mining and data fusion, efficient routing with the view of hierarchical data aggregation. The hierarchical aggregation method itself signifies beneficial function of all data networks as it decreases important energy consumption and saves bandwidth in ad hoc network. And also it makes sure of repeatability-of-tests through multiple test-environments which is a problematic task in many situations in wireless networks. Eventually the accuracy of the location identification is critical aspect of any algorithm and technique that will be the important aspect of this paper.
\end{abstract}

Keywords: Accurate node Location identification, Energy consumption, MANET, Wireless sensor networks, WSN.

\section{Introduction}

Monitoring the node is one of the imperative areas in wireless ad hoc mobile networks. The term localization in the wireless ad hoc network is basic problem for various applications of wireless sensor network. Since the sensor nodes can be embedded in many disaster areas, inaccessible terrains, and also the place and position of the wireless sensor nodes are unknown, the localization is important in many applications.

Localization in wireless networks is characterized to groups of decentralized techniques and centralized strategies. The difference is easy; in decentralized localization type, region identity is finished in an allotted way in every node separately whilst the localization inside the centralized schemes is controlled from one commonplace place of the network. In General, node localization is based totally at the sort of records which nodes can share and acquire from the alternative nodes of network. Every node within the network need to be able to get beneficial records from carried out dimension or received information. The localization techniques are divided into 2 kinds based on community verbal exchange variety; Range-aware algorithms and hop-based additionally recognized $\mathrm{s}$ variety-unfastened algorithms. Range aware algorithms estimate the gap and the route of two nodes from a measurement. Measurement techniques can be divided into 3 agencies: Angle of arrival measurements, distance associated measurements and acquired sign power profiling strategies.

Deploying the nodes in the sensor network is also crucial part and one way to deploy is to throw the nodes through the selected area. Scattering the nodes creates the network topology random. These networks are extremely instigated to execute a various chores ranging from natural habitat monitoring, environmental, home networking, medical applications and smart battle fields. There are many occurrences of research works exists and experimented huge amount of data in biological fields as well.

Upcoming two sections we will discuss summary and description of MANET localization systems and their components and then discuss about main techniques used by localization systems in order to approximation distances and angles. Subsequently we will see the best methods that can be charity by any node to calculate the position, and finally how all the estimated distance information and position information can be computed so that it allows almost every node in a MANET to find their locations. Lastly we present our conclusions about the analysis. 


\section{Literature Review}

"Distributed target localization and tracking using distributed bearings ensors" in 2016 by Lakshmi K Raju (a), Febi Ibrahim (b) P Muralikrishna (c), in this paper author discusses about Sensors, bearing measurements and position information.

"A joint routing and localization algorithm for emergency scenario" in 2012 by M. Carli (a), S. Panzieri (b), F. Pascucci (c), In this articles, the writers proposed to tackle routing and localization problems and also to reduce the network signaling that is the maximum power consuming operation in MANETs. Particularly, distributed localization algorithm is proposed based on the ranging method, designed by mapping localization into random probability distribution estimation problematic for systems with uncertainty.

"Simulation of Target Tracking in Wireless Sensor Network" in 2014, by Prof. Sachin Deshpande, Dr. J. W. Bakal (a), Prof Mritunjaykumar Ojha (b), in this paper author proposed a method known as triangulation and tri-lateration to find the target location.z

"Prediction Based Moving Object Tracking in Wireless Sensor Network", Prajakta Joshi (a), Akhila Joshi (b), in this paper author discusses about tracking the moving object in a clustered network and also about prediction mechanism to predict next location of the object.

"Location and Position Estimation in Wireless Sensor Networks", Muhammad Farooq-i-As am(a) and Muhammad Naeem Ayya (b), in this paper author discusses about various algorithms such as Single hop Active Badge, Multi-node TDoA, Multi-signal TDoA, Euclidean Propagation Method, DV-Distance Propagation Method, DV-Hop Propagation Method, Bulusu's Algorithm, Iterative and Collaborative Multi-lateration.

"An ACO-ANN based feature selection algorithm for big data", R.Joseph Manoj (a), M.D.Anto Praveena (b), K.Vijayakumar (c), in this paper authors discusses about the various approach for the choosing the subset of the given dataset based on the features available. To minimize the iteration of finding the subset is being reduced.

"Continuous security assessment of cloud based applications using distributed hashing algorithm in SDLC", K.VijayaKumar (a), C.Arun (b), in this paper authors introduced a distributed minhash algorithm to identify the similarity for for continuous security assessment.

\section{Problem Statement}

A wireless sensor network has ' $n$ ' number of nodes with the communication range 'r'. For any two nodes in the network say for example ' $x$ ' and ' $y$ ' in the network $x$ can reach $y$ only if $y$ can reach $\mathrm{x}$ by using the same level of signal strength $\mathrm{w}$. Therefore we can represent the graph as $\quad G=(V, E)$.

1. In which $\mathrm{V}=\left\{x_{1}, x_{2}, x_{3}, \ldots . x_{n}\right\} \rightarrow$ set of nodes

2. $\{\mathrm{i}, \mathrm{j}\} \in \mathrm{E}$ where $x_{i}$ reaches $x_{j}$ at the distance $\mathrm{r}$ which is the communication range.

3. $w(e)<=r ; r \rightarrow$ weight of the edge $e=\{i, j\}$

Nodes for which the location information is unknown called the unknown nodes. The ultimate aim of this paper is to find the location of these unknown nodes. The nodes at the starting stage it was unknown and later by using the localization technique the location of the node is estimated then it is called as the settled nodes. The nodes which has manual placement or the location is estimated by the GPS is called Beacon or landmark or Anchor nodes. These nodes no need to get the location information from the localization technique.

Given the network that consist of multi hop situation with the node types of anchor/beacon nodes, unidentified nodes, etc. we need to treasure the location of the unknown nodes.

\section{Proposed Statement}

The proposed localization system is classified into three main parts Angle/Distance estimation, position calculation, localization algorithm

\section{i. Angle/Distance Estimation:}

The distance and angle estimation is used to find how far the node is from the other node and the angle between and node to another node. There are many methods available to calculate the aloofness between two nodes in the network. In our system we use RSSI - Received Signal Strength Indicator.

In RSSI the distance between two nodes are found based on the received signal strength from one node to another. Sender transmits a signal to the receiver as the signal is propagated the signal strength reduces. If the receiving node is very near the strength of the signal is more and vice versa.

To find the distance between the nodes, Radio propagation classical is used. The signal strength is contrariwise proportional to the square of the distance among the nodes. But in real scenario there are many obstacles, noises etc. which makes the mathematical calculation harder. The mathematical representation of the distance calculation is given by

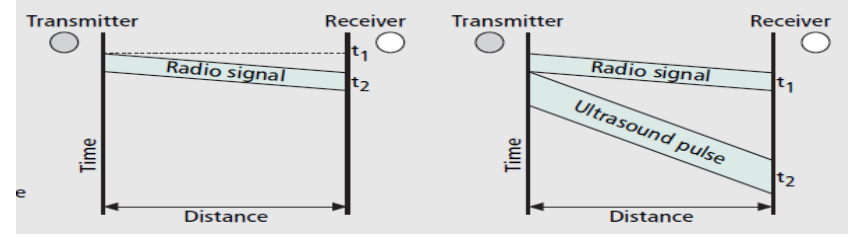

Fig. 1: Methods to find the distance between the nodes

$w \quad \alpha \frac{1}{d^{2}}$

Where $\mathrm{w}$ is the signal strength and $\mathrm{d}$ is the distance of the receiver node. Pictorial representation of the RSSI is as shown in the fig- 1 .

The angle of the node can be estimated with the help of direction of the received message. With the help of the directional antennas and the receivers we can find the angle of the node.

\section{i. Position Calculation}

When a node can find the distance and the angle from the other node, that node can calculate the position of its own. To catch the position of the node from the other node we use bounding box method

For every reference node namely $i$, a bounding box is set as a square with the center positioned as $(x i, y i)$ with sides of size $2 d i$ (here is the estimated distance) and with organizes $(x i+d i, y i+d i)$ and $(x i-d i, y i-d i)$

Connection of all the above bounding boxes can be calculated certainly without floating point operations need, by attractive the minimum of the high organizes and the maximum of the low coordinates of all bounding boxes. This is represented as sheltered rectangle in Fig. 2 given below. Eventually the absolute locus of the unidentified node is then calculated as the center of the connection of all the associated bounding boxes.

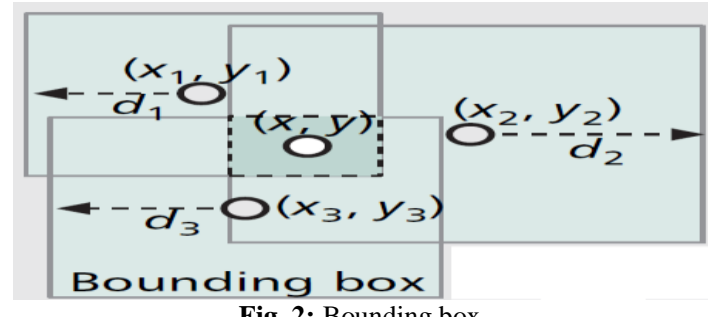

Fig. 2: Bounding box 


\section{A. Signal Strength Based Reliability}

Any node $n_{i}$ can quantity signal strength of its active neighbor-nodes. The received signal strength is unhurried at physical layer of the monitoring node in the network and the measured result can be made available access to other top layers.

\section{B. Localization Algorithm:}

The major component of the localization estimation is localization algorithm and in this algorithm we compute the exact location of the wireless sensor nodes. There are many types of localization algorithms exists they are based on,

- One hop or multi-hop

- With or without an infrastructure

- Relative or absolute locating

- distributed or centralized computation

In the proposed system we use recursive position estimation to novelty the position of the node.

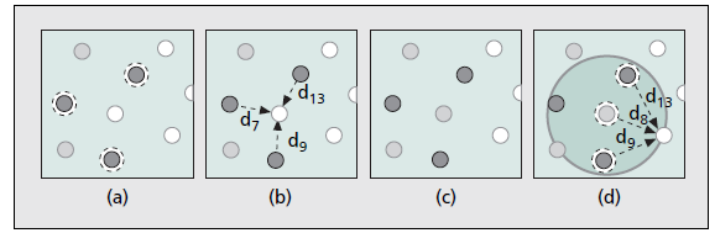

Fig. 3: MSRPE Algorithm

In the recursive position approximation we find the position of the node based on some of the initial beacon nodes. The MSRPE algorithm is further divided into four levels. At the first level the node finds the reference node, at the second phase the node calculates the distance of the reference node with the help of RSSI At the third level the node computes the position of the node using bounding box.

At final level the node itself becomes the reference node and broadcast the message to the other nodes in the network. Therefore it helps the other nodes to compute their position. Important aspect of this algorithm is that even when number of unknown nodes increases, the majority of the nodes can become the reference node and they themselves help to compute position of other unknown nodes.

\section{Algorithm: MSRPE, Multi Stage Recursive Prediction Error \\ Input : Unknown Node \\ Output : Localization of unknown node}

1. Generate nodes

2. Generate a beacon node such the position is known

3. Select a unknown node for which the location information to be found

4. Find the reference node for the unknown nodes.

5. Calculate the distance of the mysterious node from the Beacon node or reference node using RSSI

6. Calculate position of the node using bounding box algorithm

7. Record its location

8. The unknown node becomes the reference node.

9. $\operatorname{Min} \sum \mathrm{i}=\mathrm{p}+1$ to $\mathrm{n} \sum \mathrm{j} € \mathrm{Ni}\left(\mathrm{t}^{\wedge} \mathrm{ij}-\mathrm{tij}\right) 2$

10. Minimum $\sum \mathrm{i}=\mathrm{p}+1$ to $\mathrm{n}\left(\sum \mathrm{j} € \mathrm{Ni}\left(\left(\mathrm{t}^{\wedge} \mathrm{ij}-\mathrm{tij}\right) / 2\right)+\sum\left(\mathrm{t}^{\wedge} \mathrm{ij}-\mathrm{R}\right) 2\right.$

11. $E=\sum i=1$ to $n \sum j=1$ to $n(k i, j a i, j($ rij-rij 0$) 2$ ), rij $=t(i, j)$ when $i$ and $j$ are anchors

\section{Performance Analysis}

For evaluating the performance of this proposed approach, NS2 is used to make the simulation environment. Below table depicts the simulation parameters used for this performance study. DSR protocol is used with maximum of 80 mobile nodes and each node moves at an average speed of $120 \mathrm{~ms}$ randomly. Two-way-ground model is utilized and the simulation time taken is 60 seconds for each cycle of measurement. Finally the results are derived based on multiple tests with different number of nodes and different locations.

\begin{tabular}{|ll|l|}
\hline Parameter name & Parameter value & Unit \\
\hline Mobility & Two way Ground model & NA \\
Simulation Time & 60 & Seconds \\
No. of Packets & 2000 & NA \\
Application rate & 1 & mb \\
No. of Mobile Nodes & 80 & NA \\
Protocol & DSR & NA \\
Data Rate & 1 & mbps \\
Application Type & CBR & NA \\
Network Area & $1600 * 1400$ & Sq. Met \\
\hline
\end{tabular}

Fig. 4: Simulation parameters

\section{A. Latency Analysis and Result:}

Latency is described as the total time taken to deliver a packet from one node to the target node in MANET environment and it is estimated in milliseconds (ms). Below table shows the comparison of the results between the proposed system and the existing approaches.

Various node counts are considered for the performance evaluation ranging from 10 to 60 and the results are recorded as below. This data ensures the better performance in this study.

\begin{tabular}{|c|c|c|c|}
\hline \multirow{2}{*}{ Nodes } & \multicolumn{3}{|c|}{ Latency (In Milliseconds) } \\
\cline { 2 - 4 } & ADOV & SALI & Proposed System \\
\hline 10 & 26.33 & 24.54 & 23.95 \\
20 & 33.73 & 33.97 & 31.14 \\
30 & 36.35 & 34.75 & 32.11 \\
40 & 37.14 & 34.99 & 32.87 \\
50 & 42.65 & 40.55 & 39.23 \\
60 & 44.21 & 42.65 & 41.76 \\
\hline
\end{tabular}

Fig: 5: Latency- Result comparison between other systems

\section{B. Accuracy:}

From the measured result data, based on different parameters and configurations, the standard deviation in selection coordinates are computed. For instance, obtained results and the produced SDx, Standard deviation are based on various range of cognitive sets, by adjusting/in/decreasing node count by 10,20 and also by changing the speed of simulation and the standard simulation methods. The test yields more accuracy even after increasing the number of nodes. Accuracy-emphasis result is in below figure 6 and it confirms a very minimal error rate even after increasing the node count to 80 .

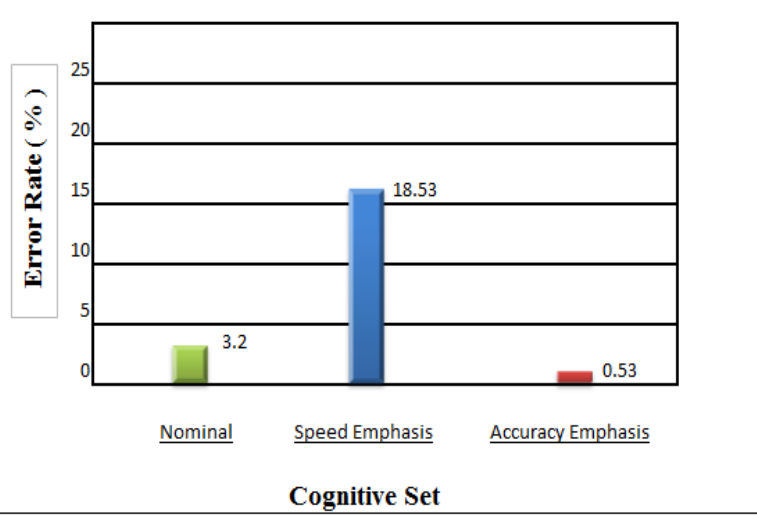

Fig: 6: Accuracy - Comparison between Speeds, Accuracy emphasis 


\section{Conclusion}

We classified localization systems into three main components they are, distance/ angle approximation, position computation, and localization algorithm. For instance, a localization system might yield enhanced results if the TDOA technique is used in its place of RSSI to estimate distances and the reverse way could also yield. Various algorithms and techniques are proposed and introduced in MANET localization study. However, each of these localization algorithms produces dissimilar level of accurateness of position identification. Localization is unswervingly related to the number of beacon nodes arranged, signal strength, routing protocol, etc. Better level of position-accuracy can be attained if a higher number of beacon nodes are arranged and it might usually need an extra piece of hardware to be auxiliary to sensor node for extent of numerous parameters such as weight, size and energy expenses. It can be determined that the NS2 simulator ropes various level of simulation for localization methods with some confines due to some physical existence of hardware setup. Because each new node is able to (after becoming the reference node), sense the other node, achieve simple computations and communicate with its further sensors, the accuracy of the localization is increased when compared to other techniques in this multi stage tracker approach. More accuracy is also because the errors are detected and banned from being propagated.

\section{References}

[1] I. F. Akyildizet al., "Wireless Sensor Networks: A Survey, "Comp. Networks, vol. 38, no. 4, Mar. 2002, pp. 393-422.

[2] Guangjie Han and Jinfang Jiang, "A Survey on Mobile Anchor Node Assisted Localization in Wireless Sensor Networks," IEEE Xplore, Mar. 2016, pp. 2220 - 2243.

[3] K. Whitehouse and D. Culler, "Calibration as Parameter Estimation in Sensor Networks," WSNA '02: Proc. 1stACM Int'l. Wksp. Wireless Sensor Networks and Apps. ACM Press, 2002, pp. 59-67.

[4] A. Savvides, C.-C. Han, and M. B. Strivastava, "Dynamic FineGrained Localization in Ad-Hoc Networks of Sensors,"7th ACM/IEEE Int'l. Conf. Mobile Computing and Networking, Rome, Italy, 2001, pp. 166-79.

[5] T. He et al., "Range-Free Localization Schemes for Large-scale Sensor Networks," MobiCom '03, ACM Press, 2003, pp. 81-95.

[6] J. Bachrach and C. Taylor, "Localization in Sensor Networks, "Handbook of Sensor Networks: Algorithms and Architectures, I. Stojmenovic, Ed., Wiley, Sept. 2005.

[7] B. Hofmann-Wellenho, H. Lichtenegger, and J. Collins, Global Positioning System: Theory and Practice, 4thed. Springer-Verlag, 1997.

[8] N. B. Priyanthaet al., "The Cricket Compass for Context-Aware Mobile Applications," 7th ACM Int'l. Conf. Mobile Computing and Networking, Rome, Italy, July 2001.

[9] Y. Fu et al., "The Localization of Wireless Sensor Network Nodes Based on DSSS," Electro/Infor. Tech., 2006IEEE Int'l. Conf., 2006, pp. 465-69.

[10] K. Whitehouse, "The Design of Calamari: An Ad HocLocalization System for Sensor Networks," Master's thesis, UC Berkeley, 2002.

[11] D. Niculescu and B. Nath, "Ad Hoc Positioning System(APS) Using AOA," Proc. INFOCOM '03, San Francisco, CA, 2003.

[12] V. Madurai and M. L. Sichitiu, "Localization in Wireless Sensor Networks: A Probabilistic Approach," Proc.ICWN 2003, Las Vegas, NV, June 2003, pp. 275-81

[13] S. Simic and S. Sastry, "Distributed localization in wireless ad hoc networks," UC Berkeley, Tech. rep.UCB/ERL M02/26, 2002

[14] P. Bahl and V. N. Padmanabhan, "Radar: An In-BuildingRF-Based User Location and Tracking System," Proc.IEEE INFOCOM 2000, vol. 2, Tel Aviv, Israel, Mar. 2000, pp. 775-84.

[15] L. Doherty, K. S. Pister, and L. E. Ghaoui, "ConvexPosition Estimation in Wireless Sensor Networks," IEEEICC '01, vol. 3, Anchorage, AK, Apr. 2001, pp. 1655-63.

[16] Y. Shang and W. Ruml, "Improved MDS-Based Localization, "IEEE ICC '04, and vol. 4, Mar. 2004, pp. 2640-51.

[17] J. Albowicz, A. Chen, and L. Zhang, "Recursive Position Estimation in Sensor Networks," 9th Int'l. Conf. Network Protocols, Nov. 2001, pp. 35-41.
[18] D. Niculescu and B. Nath, "Ad Hoc Positioning System(APS)," IEEE GLOBECOM '01, San Antonio, TX, Nov.2001, pp. 2926-31.

[19] N. B. Priyantha, A. Chakraborty, and H. Balakrishnan, "The Cricket Location-Support System," Mobile Comp.\& Networking, Boston, MA, Aug. 2000, pp. 32-43.

[20] N. Bulusu, J. Heidemann, and D. Estrin, "GPS-Less Low-cost Outdoor Localization for Very Small Devices," IEEEPers. Commun. vol. 7, no. 5, Oct. 2000, pp. 28-34.

[21] M. Arul selvan, S. Selvakumar, "Statistical approach location identification in MANET" in 2015, I J C T A, 8(4), 2015, pp. 1521 1525

[22] S. Capkun, M. Hamdi, and J.-P. Hubaux, "GPS-Free Positioning in Mobile Ad Hoc Networks," Cluster Computing, vol. 5, no. 2, 2002, pp. $157-67$.

[23] M. L. Sichitiu and V. Ramadurai, "Localization of Wireless Sensor

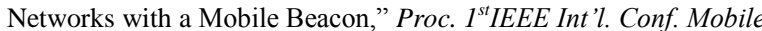
Ad Hoc and Sensor Sys., FL, Oct. 2004, pp. 174-83.

[24] R.Joseph Manoj, M.D.Anto Praveena, K.Vijayakumar, "An ACOANN based feature selection algorithm for big data", Cluster Computing the Journal of Networks, Software Tools and Applications, ISSN: 1386-7857 (Print), 1573-7543 (Online) DOI: 10.1007/s10586-018-2550-z, 2018

[25] K. Vijayakumar C. Arun, "Continuous security assessment of cloud based applications using distributed hashing algorithm in SDLC", Cluster Computing DOI 10.1007/s10586-017-1176-x, Sept 2017.

[26] K. Vijayakumar, C.Arun, Automated risk identification using NLP in cloud based development environments Ambient Intell Human Computing, DOI 10.1007/s12652-017-0503-7, Springer May 2017. 